Exercices Exercices de rhétorique

de rhétorique $\quad 8 \mid 2017$

La rhétorique biblique et sémitique

\title{
Une nouvelle présentation de la rhétorique biblique et sémitique
}

\section{Roland Meynet}

\section{OpenEdition}

1 Journals

Édition électronique

URL : https://journals.openedition.org/rhetorique/486

DOI : 10.4000/rhetorique.486

ISSN : 2270-6909

Éditeur

UGA Éditions/Université Grenoble Alpes

Édition imprimée

ISBN : 978-2-84310-343-8

\section{Référence électronique}

Roland Meynet, "Une nouvelle présentation de la rhétorique biblique et sémitique », Exercices de rhétorique [En ligne], 8 | 2017, mis en ligne le 25 janvier 2017, consulté le 28 novembre 2021. URL http://journals.openedition.org/rhetorique/486 ; DOI : https://doi.org/10.4000/rhetorique.486

Ce document a été généré automatiquement le 28 novembre 2021.

\section{(c) (1) () (2)}

Les contenus de la revue Exercices de rhétorique sont mis à disposition selon les termes de la Licence Creative Commons Attribution - Pas d'Utilisation Commerciale - Partage dans les Mêmes Conditions 4.0 International. 


\title{
Une nouvelle présentation de la rhétorique biblique et sémitique
}

\author{
Roland Meynet
}

1 «Vous avez dit: "Nouvelle"?». Oui, parce qu'une ancienne ${ }^{1}$ est disponible depuis longtemps, et en plusieurs langues.

2 La rhétorique classique, gréco-latine existe; preuve en est la multitude de traités de rhétorique qui ont fleuri depuis Aristote jusqu'à nos jours. La rhétorique classique pourtant n'est pas la seule au monde. En dehors du monde grec, puis latin, puis occidental, on discourait et on écrivait aussi, bien avant le IV ${ }^{\mathrm{e}}$ siècle avant J.-C. En ce qui concerne la Bible et, plus largement, le monde sémitique, la manière de s'exprimer, oralement ou par écrit, est fort différente de celle du monde gréco-latin. Contrairement à ce dernier, la littérature hébraïque antique n'a pas laissé le moindre manuel de rhétorique. Dès les premiers siècles de notre ère, les premiers commentateurs de la Bible, les Pères grecs ou latins, tels Origène, Jérôme ou Augustin, ont donc abordé ces textes avec les outils qu'ils connaissaient et ils s'ingéniaient à trouver dans la poésie hébraïque, en particulier dans les Psaumes, pentamètres, hexamètres ou autres mètres de la poésie qu'ils avaient étudiée durant leur formation classique.

Ce n'est donc pas par des traités laissés par les anciens que l'on sait qu'il existe une rhétorique biblique et sémitique, différente de la gréco-latine. Il a fallu la découvrir, petit à petit, par l'étude des textes eux-mêmes. Et c'est avec ceux de la Bible que tout a commencé. Une des premières choses que l'on mentionne pour définir l'identité d'une personne, d'une nation, d'une école, est sa date de naissance. On dit communément que la rhétorique biblique est "née " en 1753. Même si ce n'est pas vrai, c'est commode. Cette année-là Robert Lowth publie ses Leçons sur la poésie sacrée des Hébreux qu'il professait à Oxford. C'est à lui qu'on attribue la découverte du parallélisme des membres. En bref, il distingue trois sortes de parallélismes ${ }^{2}$ :

1. le parallélisme synonymique

«Quand Israël sortit d'Égypte,

la maison de Jacob d'un peuple barbare » (Ps 114,1) 
2. le parallélisme antithétique

«Les blessures de l'ami sont fidèles

mais les baisers de l'ennemi sont trompeurs » $(\operatorname{Pr} 27,6)$

3. le parallélisme synthétique ou de composition

Ce sont tous les parallélismes qui n'entrent pas dans les deux premières catégories.

Lowth a aussi montré que, à côté des distiques, tels ceux qui viennent d'être cités (que nous appelons « segments bimembres »), il existe aussi des " segments trimembres » :

Les flots ont élevé, Seigneur,

Les flots ont élevé leur voix,

Les flots ont fait entendre leurs mugissements (Ps 93,3)

et même, quoique beaucoup moins fréquents, des « unimembres ». Il les découvre dans ce qu'il appelle « les périodes à cinq membres » :

C'est ainsi que rugit le lion,

Et le lionceau, sur sa proie ;

Les bergers se rassemblent en foule contre lui ;

À leur voix il ne s'épouvantera point,

Et à leurs cris tumultueux son courage ne sera point abattu (Is 31,4).

6 Avec ces trois sortes de "segments ", bimembres, trimembres ou même unimembres, est déjà mis en place un des fondements de la rhétorique biblique, le segment représentant le premier niveau de composition des textes.

7 Une dernière chose doit être soulignée : Lowth dit lui-même qu'il n'est pas l'inventeur du parallélisme des membres. Il se réfère longuement en effet à Azarias dei Rossi, un rabbin italien du XVI siècle qui avait déjà montré la spécificité de la poésie hébraïque par rapport à celle du monde occidental (voir la traduction française de la $19^{\mathrm{e}}$ leçon de Lowth ${ }^{3}$.

8 Si Lowth n'a pas été le premier, il n'a pas été non plus le dernier. Il ne fut en réalité qu'un précurseur. Ce n'est que dans les années 1820 qu'apparurent ceux que l'on peut considérer comme les véritables fondateurs de ce qui s'appelle désormais la rhétorique biblique; Thomas Boys (1824 et $\left.1825^{4}\right)$ mais surtout John Jebb $\left(1820^{5}\right)$ auquel est consacrée la dernière rubrique du présent numéro d'Exercices de rhétorique ${ }^{6}$.

Un homme qui vit dans la Jérusalem d'autrefois ou dans quelque autre ville d'Israël longtemps avant notre ère donne à ses paroles un tour et une suite, un style qui n'est pas le nôtre. S'y habituer est l'une des plus grandes joies que donne l'exégèse. Mais cette joie demande quelque patience. (Paul Beauchamp, Psaumes nuit et jour, Paris, Le Seuil, 1980, p. 155)

La rhétorique biblique existait, mais il a fallu la découvrir, par l'examen des textes euxmêmes. Et cela a pris du temps, beaucoup de temps. Voilà qui est tout à fait dans la manière biblique. Une formule, à l'emporte-pièce, dit la différence fondamentale entre les deux rhétoriques, la classique et la biblique : « Le Grec démontre, le Juif montre ». Le Grec veut convaincre, par des preuves et des raisonnements, le Juif indique le chemin que le lecteur peut emprunter s'il désire comprendre. Pour forcer le trait, la rhétorique occidentale entend contraindre l'auditeur à adopter son opinion et son jugement, la biblique respecte la liberté du lecteur, sa responsabilité, sa dignité.

La rhétorique biblique peut être définie par ses deux caractéristiques fondamentales : la binarité et la parataxe. La binarité, parce que les choses sont dites deux fois. On l'a déjà entraperçu avec le segment bimembre synonymique, mais la binarité se retrouve à tous les niveaux de l'organisation textuelle : il existe deux récits de la création (Gn 1 et Gn 2); deux décalogues (Ex 20 ; Dt 5); deux récits de l'enfance de Jésus (selon Matthieu, 
selon Luc), deux «Notre Père» (Mt 6; Lc 11), finalement, pour les chrétiens, deux Testaments, l'Ancien et le Nouveau.

11 La parataxe, c'est que les deux choses qui vont ensemble sont simplement juxtaposées, et que c'est au lecteur de saisir leur rapport. Comment tenir ensemble les deux récits de la création, qui semblent tellement différents, et que signifie leur mise en parallèle? Pourquoi donc deux décalogues? Voilà qui invite à réfléchir?

Poussant davantage la réflexion, il apparaît que binarité et parataxe sont un magnifique exemple 1) de binarité, car les caractéristiques de la rhétorique biblique sont deux, et 2) de parataxe, car rien n'a été dit jusqu'ici sur le rapport entre binarité et parataxe ! Voilà une belle énigme ! Eh bien, justement, la caractéristique fondamentale de la rhétorique biblique est une seule : son caractère énigmatiques.

13 Jusqu'ici on a parlé de rhétorique biblique, sans distinguer entre Ancien et Nouveau Testament. Or si ce qu'on appelle la Bible hébraïque a été écrite dans deux langues sémitiques, essentiellement l'hébreu, mais aussi l'araméen, la Bible des premiers chrétiens, qui était la Bible grecque des Septante (traduction de la bible hébraïque faite par les Juifs au $\mathrm{II}^{\mathrm{e}}$ siècle avant notre ère en Égypte), comprenait aussi quelques livres rédigés en grec, comme le livre de la Sagesse de Salomon, ou transmis en grec, comme Ben Sira (dit aussi le Siracide ou l'Ecclésiaste), qu'on appelle les « deutérocanoniques ». Quant au Nouveau Testament, tous ses livres ont été transmis en grec. Or, comme les auteurs des deutérocanoniques, les auteurs du Nouveau Testament étaient tous des Juifs, ou pour le moins des auteurs tellement imbibés de la bible juive, qu'ils composaient en suivant les lois de composition des textes de ce que nous appelons l'Ancien Testament, mais qui pour eux étaient tout simplement « les Écritures ».

14 Il faut ajouter que l'étude de quelques textes d'autres littératures du monde sémitique antique, akkadien, ougaritique, égyptien pharaonique, etc., ou plus récents, comme le Coran et les Hadiths, conduit à parler de rhétorique sémitique et non seulement biblique.

15 Ajoutons encore que des auteurs qui, comme les auteurs juifs du Nouveau Testament, n'écrivent pas dans une langue sémitique, mais en latin, en paléoslave, en français, composent à la manière biblique. Saint Benoît dans sa Règle, saint Clément d'Okrid dans ses hymnes, Pascal dans les Pensées, sont tellement imprégnés de Bible qu'ils composent - sans même s'en rendre compte probablement - selon les lois de la rhétorique sémitique ${ }^{9}$

16 Contrairement à la plupart des publications modernes, les livres bibliques - à part celui des Psaumes - ne fournissent pas de divisions internes. Les seules limites indiscutables sont le début et la fin du livre ! D'où l'importance que l'analyse rhétorique biblique a dû accorder à la distinction des niveaux d'organisation des textes, pour lesquels une terminologie spécifique a été élaborée peu à peu. On distingue deux grandes catégories de niveaux :

\footnotetext{
1 - LES NIVEAUX DE COMPOSITION

1.1. - Les niveaux « inférieurs" (ou non autonomes) ou " de citation »

À part les deux premières (le terme et le membre), les unités de niveau inférieur sont formées d'une, deux ou trois unités du niveau précédent.
} 
Terme

Le terme correspond en général à un «lexème ", ou mot qui appartient au lexique : substantif, adjectif, verbe, adverbe.

Membre

Le membre est un syntagme, ou groupe de "termes » liés entre eux par des rapports syntaxiques étroits. Le « membre » est l'unité rhétorique minimale ; il peut arriver que le membre comporte un seul terme (le terme d'origine grecque est « stique »).

Segment

Le segment comprend un, deux ou trois membres ; on parlera de segment « unimembre » (le terme d'origine grecque est «monostique »), de segment «bimembre » (ou «distique ») et de segment « trimembre » (ou « tristique »).

Morceau

Le morceau comprend un, deux ou trois segments.

Partie

La partie comprend un, deux ou trois morceaux.

\section{2. - Les niveaux " supérieurs" (ou autonomes) ou " de récitation"}

Ils sont tous formés soit d'une, soit de plusieurs unités du niveau précédent.*

Passage

Le passage - l'équivalent de la « péricope » des exégètes - est formé d'une ou de plusieurs parties.

Séquence

La séquence est formée d'un ou de plusieurs passages.

Section

La section est formée d'une ou de plusieurs séquences.

Livre

Le livre est formé d'une ou de plusieurs sections.

Il est quelquefois nécessaire d'avoir recours aux niveaux intermédiaires de la « sous-partie », de la "sous-séquence » et de la "sous-section »; ces unités intermédiaires ont la même définition que la partie, la séquence et la section ${ }^{10}$.

\section{2 - LES FIGURES DE COMPOSITION}

À chacun de ces niveaux, les textes sont organisés selon ce qu'on appelle des « figures ». Le système est très simple. On distingue :

2.1. - Les symétries totales 
Construction parallèle

Figure de composition où les unités en rapport deux à deux sont disposées de manière parallèle : $\mathrm{A}$ B C D E | A'B'C'D'E'.

Quand deux unités parallèles entre elles encadrent un élément unique, on parle de parallélisme pour désigner la symétrie entre ces deux unités, mais on considère l'ensemble (l'unité de niveau supérieur) comme une construction concentrique : A $|\mathrm{x}| \mathrm{A}^{\prime}$.

Pour « construction parallèle », on dit aussi « parallélisme » (qui s'oppose à « concentrisme »).

Construction spéculaire

Figure de composition où les unités en rapport deux à deux sont disposées de manière antiparallèle ou « en miroir » : A B C D E | E'D'C'B'A'.

Comme la construction parallèle, la construction spéculaire n'a pas de centre; comme la construction concentrique, les éléments en rapport se correspondent en miroir.

Quand la construction ne comprend que quatre unités, on parle aussi de « chiasme »: A B | B'A'.

Construction concentrique

Figure de composition où les unités symétriques sont disposées de manière concentrique : $\mathrm{A} \mathrm{B} \mathrm{C} \mathrm{D} \mathrm{E}$ $|\mathrm{x}| \mathrm{E}^{\prime} \mathrm{D}^{\prime} \mathrm{C}^{\prime} \mathrm{B}$ 'A', autour d'un élément central (cet élément peut être une unité de l'un quelconque des niveaux de l'organisation textuelle).

Pour "construction concentrique", on peut dire aussi "concentrisme" (qui s'oppose à « parallélisme »).

\section{2. - Les symétries partielles}

Termes initiaux

Termes ou syntagmes identiques ou semblables qui marquent le début d'unités textuelles symétriques ; l'« anaphore » de la rhétorique classique.

Termes finaux

Termes ou syntagmes identiques ou semblables qui marquent la fin d'unités textuelles symétriques ; l'« épiphore » de la rhétorique classique.

Termes extrêmes

Termes ou syntagmes identiques ou semblables qui marquent les extrémités d'une unité textuelle ;

l'« inclusion » de l'exégèse traditionnelle.

Termes médians

Termes ou syntagmes identiques ou semblables qui marquent la fin d'une unité textuelle et le début de l'unité qui lui est symétrique; le «mot-crochet» ou "mot-agrafe » de l'exégèse traditionnelle.

Termes centraux

Termes ou syntagmes identiques ou semblables qui marquent les centres de deux unités textuelles symétriques.

On trouvera sur le site de la RBS la terminologie en différentes langues ${ }^{11}$. 
L'analyse rhétorique biblique a développé tout un art de réécrire le texte pour donner à voir sa composition. Voici les principales règles de réécriture:

- à l'intérieur du membre, les termes sont généralement séparés par des blancs ;chaque membre est généralement réécrit sur une seule ligne;

- les segments sont séparés par une ligne blanche ;

- les morceaux sont séparés par une ligne discontinue ;

- la partie est délimitée par deux filets ; il en va de même pour les sous-parties.

- à l'intérieur du passage, les parties sont encadrées (sauf si elles sont très courtes, comme une introduction ou une conclusion) ; les éventuelles sous-parties sont disposées dans des cadres contigus ;

- à l'intérieur de la séquence ou de la sous-séquence, les passages, réécrits en prose, sont disposés dans des cadres séparés par une ligne blanche ;

- à l'intérieur de la séquence, les passages d'une sous-séquence sont disposés dans des cadres contigus.

- Ces règles seront suivies, bien évidemment, dans toutes les analyses de texte de ce numéro d'Exercices de rhétorique ${ }^{12}$.

Sur les lois de composition des textes bibliques, l'essentiel vient d'être dit à propos des différents types de «symétries ». Il vaut cependant la peine d'en mentionner d'autres. Il arrive souvent que le centre des compositions concentriques soit occupé par une question (on parlera donc de « la loi de la question au centre»), par une citation («la loi de la citation au centre »). Le centre peut être aussi un proverbe; il est en somme quelque chose qui fait problème, et, pour utiliser un terme déjà employé, qui est «énigmatique ». Le centre d'un texte en est la clé de voûte, donnant cohérence à l'ensemble, articulant tous les autres éléments ; c'en est donc la clé de lecture. L'image biblique la plus parlante des constructions concentriques est la menorah, le chandelier à sept branches. La branche centrale est celle par laquelle tout tient, ce qui n'est pas du tout le cas des poupées russes auxquelles on a voulu comparer ce genre de composition.

Un des reproches qui est souvent fait aux praticiens de l'analyse rhétorique biblique, c'est ce qu'un auteur a appelé «la maladie du chiasme » (on emploie à tort ce terme pour parler des compositions concentriques). Ils verraient des chiasmes partout. Il est vrai qu'il y en a beaucoup, mais ce n'est pas de la faute de ceux qui les découvrent et les décrivent; s'il y a beaucoup de phrases nominales dans les textes hébreux, ce n'est pas de la faute des grammairiens ! À côté des compositions concentriques, il existe aussi des textes construits en parallèle et d'autres de manière spéculaire.

À ce propos, il faut rappeler que ces deux derniers types de composition n'ont pas de centre. Alors, comment faire pour ouvrir leur sens, puisqu'ils sont dépourvus de la « clé de lecture » que représente le centre des compositions concentriques? On a parlé, à juste titre, de "centre absent ${ }^{13}$ ». L'énigme dans ce cas est redoublée : l'artiste doit travailler sans filet. Il se trouve dans la situation, fort inconfortable, de Daniel, quand Nabuchodonosor, roi de Babylone, lui demande non seulement d'interpréter son rêve, mais d'abord de le lui raconter (Dn 2)!

Découvrir la composition du texte ne suffit pas. Un texte ne se comprend vraiment que dans son contexte. Celui-ci peut être proche ou lointain. Le premier contexte de ce que nous appelons un " passage » (les exégètes disent souvent une " péricope ", c'est-à-dire, dans les évangiles, un récit de guérison, une parabole, un court discours), c'est la "séquence » à laquelle il appartient. Ainsi le passage de "La Visitation » (Lc 1,39-45) fait partie d'une séquence (Lc 1,26-55) qui comprend aussi « L'Annonciation » (28-38) et «Le cantique de Marie» (46-55), le tout précédé d'une courte «Introduction » (26-27) et suivi d'une conclusion plus courte encore (56).

22 Toutefois, le contexte peut être fort éloigné, mais n'en est pas moins capital pour comprendre le texte. Ainsi le passage central de la séquence de Luc dont il vient d'être 
question, «La Visitation », est focalisé sur une question qui rappelle celle que David avait prononcée quand il avait fait venir l'arche d'alliance à Jérusalem (en traduction littérale $\left.{ }^{14}\right)$ :

\begin{tabular}{|l|l|l|l|l|l|l|}
\hline Comment & l'arche & DU & SEIGNEUR & VIENDRA & À MOI ? & (2S 6,9) \\
\hline D'où à moi que & la mère & DE mon & SEIGNEUR & SOIT VENUE & À MOI ? & (Lc 1,43) \\
\hline
\end{tabular}

Composition et contexte conduisent à l'interprétation. Il est bien évident que tout le travail d'analyse, la division des textes à leurs différents niveaux, le repérage des figures de composition, la mise en évidence du contexte - sans parler des opérations préliminaires de la critique textuelle, de la résolution des problèmes grammaticaux, des enquêtes de lexicographie -, tout cela n'a qu'une seule fin : comprendre et expliquer le texte, découvrir son sens.

De même qu'ont été énoncées les lois de composition des textes, ainsi s'est-on risqué à proposer «Cinq lois herméneutiques » qui s'énoncent de la manière suivante :

1. Chercher la différence: quand deux unités semblent identiques, cherchez la différence.

2. Chercher la ressemblance: quand deux unités en rapport semblent opposées à tous les égards, cherchez la ressemblance.

3. Partir du centre: dans les constructions concentriques le centre est la clé de lecture.

4. Suivre le fil rouge : l'image du «fil rouge » traduit ce qu'on pourrait appeler «le principe de cohérence ».

5. Croiser les fils: cette image traduit ce qu'on pourrait appeler «le principe d'articulation".

Il va sans dire que, bien souvent, ces lois jouent ensemble ${ }^{15}$.

On a dit en commençant que les anciens commentateurs chrétiens de la Bible cherchaient dans ses textes poétiques des pentamètres et des hexamètres. En manière d'« inclusion sémitique ", revenons sur la question, avant de présenter le contenu du fascicule de la présente revue. Répondant aux critiques païennes qui se moquaient de la rusticitas des textes bibliques, ils expliquaient que c'était là une manifestation de l'humilitas de Dieu. Ce faisant, ils admettaient que les textes bibliques ne pouvaient pas entrer en compétition avec la littérature grecque puis latine. Jusqu'à nos jours, beaucoup pensent encore que les livres bibliques ne sont pas des œuvres littéraires, mais des productions populaires.

Cela n'a pas empêché les commentateurs de se servir des catégories et des règles de la rhétorique classique pour analyser les textes bibliques. Beaucoup le font encore de nos jours. Il est hors de question de nier que l'on puisse obtenir ainsi quelque résultat. Toutefois, on se demandera si c'est là le meilleur outil pour comprendre et expliquer des textes qui ne relèvent pas de la même aire culturelle que les littératures grecque et latine.

À la fin du discours de Jésus dans la plaine, Luc place ce qu'il présente comme « une parabole ", au singulier. Pour un esprit occidental, une parabole est un court récit, comme la fameuse parabole dite du fils prodigue. Or, pour lui, la parabole de Lc 6,39-49 a toutes les apparences d'un pot-pourri : il s'agit en effet de deux aveugles, puis de la paille et de la poutre dans l'œil, après quoi on saute sans crier gare à l'arbre et son fruit, bon ou malade, puis ce sont les ronces et les épines avec les figues et le raisin et cela 
finit avec les deux maisons, l'une fondée sur le roc, l'autre bâtie sur la terre sans fondement. Selon le grand spécialiste américain de la rhétorique classique, George A. Kennedy, "ce n'est pas un très bon discours ». Alors que Luc utilise le singulier, Kennedy considère ces onze versets comme "des paraboles ", ce qui montre bien qu'il n'en saisit pas l'unité. Il ajoute que «ces [paraboles] aux versets 39-40 sont passablement obscures ", que, pour les versets $43-45$, «les prémisses ne sont pas arrangées de façon très logique. L'efficacité de l'ensemble s'en trouve par conséquent amoindrie. Le verset 46 est une récapitulation obscure ${ }^{16} »$.

La parabole lucanienne n'est pas un patchwork, c'est une tapisserie dont les motifs s'entrelacent selon une logique qui, pour être différente de la nôtre, n'en est pas moins fort rigoureuse. En bref, il s'agit tout au long du disciple de Jésus devenu maître à son tour. S'il n'y voit pas, parce qu'il est aveugle ou qu'il a une poutre dans l'œil, il ne pourra pas guider ou corriger son disciple. Il serait comme un arbre malade dont le fruit n'est pas bon. Mais attention, la parabole ne dit pas que c'est une affaire de nature qu'on ne pourrait changer: «les chiens ne font pas des chats». La conversion est possible : «Enlève d'abord la poutre de ton œil, et tu verras clair pour enlever la paille qui est dans l'œil de ton frère ». Pour savoir si un arbre est bon, il suffit de regarder son fruit. Mais comment le disciple saura si son maître est bon? D'abord, si ses actes s'accordent avec ses paroles; ensuite et surtout s'il tient bon dans la tempête de l'épreuve ${ }^{17}$.

"Qui n'est jamais sorti de son village croit que seule sa mère sait faire la bouillie " (proverbe africain). La manière de composer des auteurs bibliques, et plus largement sémitiques, n'est pas la même que celle de la rhétorique occidentale. Attention à l'ethnocentrisme! Les commentateurs de la Lettre aux Galates qui utilisent la rhétorique classique, à commencer par Hans-Dieter Bet $^{18}$ lequel se réfère constamment à Cicéron et Quintilien, vont donc chercher à identifier la propositio principale de la Lettre. Certains la trouvent à la fin de l'exordium, soit en 1,11-12 :

${ }^{11} \mathrm{Je}$ vous fais savoir, frères, que l'Évangile que j'ai annoncé n'est pas selon un homme : ${ }^{12}$ car moi non plus ce n'est pas d'un homme que je l'ai reçu ou appris, mais par une révélation de Jésus Christ [...]

d'autres à la fin de la narratio, en 2,19-20 :

${ }^{19}$ Car moi, par la Loi pour la Loi je suis mort ; afin de vivre pour Dieu, je suis crucifié avec le Christ ; ${ }^{20}$ et ce n'est plus moi qui vis, mais le Christ qui vit en moi. Ce que maintenant je vis dans la chair, je le vis dans la foi au Fils de Dieu qui m'a aimé et s'est livré lui-même pour moi.

31 Analysée en fonction des lois de la rhétorique sémitique, la Lettre aux Galates est construite de manière concentrique et la courte séquence centrale, le cœur de l'écrit, se trouve en 3,26-29:

${ }^{26}$ Tous en effet vous êtes fils de Dieu par la foi dans le Christ Jésus ;

${ }^{27}$ car vous tous qui avez été baptisés dans le Christ, vous avez revêtu le Christ. ${ }^{28}$ Il n'y a pas de Juif ni de Grec, il n'y a pas d'esclave ni de libre, il n'y a pas d'homme et de femme.

Tous en effet vous êtes un seul dans le Christ Jésus ;

${ }^{29}$ et si vous êtes du Christ, alors vous êtes la descendance d'Abraham, héritiers selon la promesse. tenait à utiliser la terminologie de la rhétorique classique, la propositio principale. 
L'examen du contenu des trois "propositiones" mentionnées laisse percevoir que le sujet ou l'enjeu de la Lettre n'est pas du tout le même suivant les commentateurs ${ }^{19}$.

\section{ANNEXES}

\section{PRÉSENTATION DE LA LIVRAISON}

\section{DOSSIER}

Le dossier comprend quatre contributions :

1. Jacek Oniszczuk présente l'analyse d'un " passage », celui de la femme adultère (Jn $7,53-8,11)$.

2. Germano Lori et Francesco GiosuèVoltaggio, de laDomus Galileae en Israël, proposent leur analyse de la séquence du troisième chapitre de la Genèse, qui comprend cinq « passages »; ils l'ont intitulée «

La sentence de Yhwh comme chemin de rédemption et deuxième possibilité de conversion».

3. Roberto Di Paolo analyse le chapitre 10 de Matthieu ; après un court passage introductif, cette « séquence » comprend cinq passages articulés en trois sousséquences. Elle est intitulée " Le maître et le disciple envoyés à Israël et aux nations ».

4. Michel Cuypers offre l'analyse de la Sourate 69 du Coran, qui est de la taille d'un " passage » formé de trois « parties ».

\section{ATELIER}

L'atelier regroupe un article de méthodologie, puis deux longs récits :

1. Roland Meynet propose d'abord un texte où il explique «Comment réaliser des exercices d'analyse rhétorique biblique ».

2. Il raconte ensuite comment il a découvert la composition des psaumes des montées : «Les psaumes des montées (Ps 120-134) forment-ils un ensemble structuré ? Récit d'une recherche et d'une... découverte ».

3. Francesco Graziano à son tour fait le récit de sa recherche et de sa découverte de la composition de l'ensemble de l'évangile de Matthieu.

\section{DOCUMENT}

En 1820 John Jebb, ecclésiastique irlandais publiait le résultat d'une longue recherche, menée dans le sillage de Robert Lowth. De larges extraits de son ouvrage, trop longtemps oublié, sont traduits en français (puis donnés aussi dans la langue originale). 
Ce $n^{\circ} 8$ de la revue Exercices de rhétorique a été réalisé par la «Société internationale pour l'étude de la Rhétorique Biblique et Sémitique " (RBS, www.retoricabiblicaesemitica.org). Les contributions de Roberto Di Paolo, de Francesco Graziano, de Germano Lori et Francesco Giosuè Voltaggio, de Jacek Oniszczuk, toutes rédigées en italien, ont été traduites par Roland Meynet, qui a aussi traduit de l'anglais les textes de John Jebb.

\section{NOTES}

1. Voir http://www.retoricabiblicaesemitica.org/arb_articolo_fr.html.

2. Les traductions des textes bibliques de la 19e leçon de Lowth sont celle de Sicard: R. Lowth, Leçons sur la poésie sacrée des Hébreux traduites pour la première fois en français du latin du Dr Lowth, trad. M. Sicard, I-II, Lyon, Ballanche, 1812.

3. Voir http://www.retoricabiblicaesemitica.org/Lowth_fr.html.

4. T. Boys, Tactica Sacra. An Attempt to develope, and exhibit to the eye by tabular arrangements, a general rule of composition prevailing in the Holy Scriptures, Londres, T. Hamilton, 1824 ; id., A Key to the Book of the Psalms, Londres, L.B. Seeley \& Sons, 1825. Sur T.Boys, voir http:// www.retoricabiblicaesemitica.org/Boys_it.html.

5. J. Jebb, Sacred Literature, Londres, T. Cadell \& W. Davies, 1820. Sur J. Jebb, voir http:// www.retoricabiblicaesemitica.org/Jebb_it.html.

6. Voir également R. Meynet, Traité de rhétorique biblique, Pendé, Gabalda (Rhétorique Sémitique 11), 2013, chap. 1, «Historique ", p. 31-110 ; URL : http://www.retoricabiblicaesemitica.org/ RhSem_11_fr.html. Pour les contemporains, voir id., «La rhétorique biblique et sémitique. État de la question ", Rhetorica 28 [2010], p. 290-312.

7. Sur binarité et parataxe, voir R. Meynet, Traité, op. cit., p. 15-26.

8. Voir également R. Meynet, «Rhétorique biblique, rhétorique de l'énigme », Rhetorica 33 [2015], p. $147-180$.

9. À ce sujet on consultera les Actes des colloques de la RBS (Société Internationale pour l'étude de la Rhétorique Biblique et Sémitique), publiés par les EDB (Bologne, Edizioni Dehoniane Bologna, URL : http://www.retoricabiblicaesemitica.org/retorica_biblica_fr.php) puis par la G\&BPress (Rome, Gregorian \& Biblical Press, URL: http://www.retoricabiblicaesemitica.org/ retorica_biblica_semitica_fr.php).

10. Voir Traité, op. cit., chap. 3, « Les niveaux de composition », 131-215.

11. Voir ibid., « Les figures de composition », 217-281.

12. Sur les règles de réécriture, voir R. Meynet, Traité, op. cit., chap. 5, p. 283-344. Pour la réécriture des tableaux synoptiques, voir ibid., chap. 9, p. 471-506.

13. P. Bovati, «Il centro assente. Riflessioni ermeneutiche sul metodo dell'analisi retorica (biblica), in riferimento specifico alle strutture prive di centro ", R. Meynet - J. Oniszczuk, éd., Retorica Biblica e Semitica 1. Atti del primo convegno RBS, Bologne, EDB (Retorica Biblica 12), 2009, p. 107-121 ; URL : http://www.retoricabiblicaesemitica.org/ReBib_12_fr.php.

14. Voir R. Meynet, L'Évangile de Luc, Pendé, Gabalda (Rhétorique Sémitique 8), 2011, p. 72-75 ; URL : http://www.retoricabiblicaesemitica.org/RhSem_08_fr.html. Sur le contexte, voir id., Traité, op. cit., deuxième partie, « Le contexte », p. 345-506.

15. Ces cinq lois sont largement exemplifiées dans le Traité (ibid., p. 551-590). Sur l'interprétation, voir ibid., troisième partie, «L'interprétation », p. 507-635.

16. G. Kennedy, New Testament Interpretation through Rhetorical Criticism, Chapel Hill - Londres, University of North Carolina Press, 1984, p. 67 ; je traduis. 
17. Voir R. Meynet, "Histoire de l'analyse rhétorique en exégèse biblique », Rhetorica 8 [1990], p. 291-320 : toute la deuxième partie de l'article est consacrée à l'analyse rhétorique biblique de la parabole de Luc. Analyse accessible aussi dans mon commentaire de Luc, URL: http:// www.retoricabiblicaesemitica.org/RhSem_08_fr.html.

18. H.-D. Betz, Galatians: A Commentary on Paul's Letter to the Churches in Galatia, Philadelphie, Fortress, 1979 (rééd. 1984).

19. Voir R. Meynet, La lettre aux Galates, Pendé, Gabalda (Rhétorique Sémitique 10), 2012 ; URL : http://www.retoricabiblicaesemitica.org/RhSem_10_fr.html.

\section{AUTEUR}

\section{ROLAND MEYNET}

Professeur émérite de théologie biblique à la Faculté de théologie de l'Université Grégorienne de Rome, membre fondateur et Secrétaire de la RBS 